\title{
Informed consent: ethical, legal, and medical implications for doctors and patients who participate in randomised clinical trials
}

\author{
CANCER RESEARCH CAMPAIGN WORKING PARTY IN BREAST CONSERVATION
}

Since Austin Bradford Hill's seminal paper on medical ethics and controlled trials 20 years ago $^{1}$ the issue of informed consent, particularly as it relates to controlled clinical trials, has not attracted much public attention in British medical practice. In 1982, however, the coroner at an inquest on an elderly woman expressed the view that "the whole idea of concealed controlled trials [should] be brought to the public notice for proper discussion." The patient, who had cancer of the bowel, died two weeks after having been entered as an unwitting participant into a randomised clinical trial testing the efficacy of a new drug treatment.

For the past two years the Cancer Research Campaign Working Party in Breast Conservation has been devising a protocol for a trial in "early" breast cancer designed to determine whether local tumour excision, with breast conservation plus radical radiotherapy, gives results comparable with simple mastectomy plus radical radiotherapy in terms of recurrence and survival. ${ }^{2}$ This trial has now been accepted for funding by the Cancer Research Campaign and was launched on 18 November 1982. Throughout the planning period, the question of informed consent was under constant discussion.

It was agreed from the outset that a large number of patients (about 2000) would have to be entered into the trial so that it would be sensitive enough to detect differences in therapeutic

Members of the working party were: Ms Carolyn Faulder, Professor M J Peckham (chairman), Professor M Baum, Dr J A Bullimore, Dr J Cuzick, Dr K R Durrant, Dr G P Maguire, Dr J Sloane, Mr C Teasdale, Dr J S Tobias (secretary), and $\mathrm{Mr} \mathrm{H}$ White.

Correspondence and requests for reprints to: The Coordinator, CRC Breast Conservation Trial, CRC Clinical Trials Centre, Rayne Institute, 123 Coldharbour Lane, London SE5 9NU. benefit of the order of $10 \%$ with confidence. The conflict arising was, quite simply, that informed consent might result in the loss of patients to the trial. We believed that this loss could occur at two levels: firstly, the seeking of informed consent might well deter surgeons from participating in the trial and, secondly, having recruited participating surgeons, the possibility of patients who had been "fully informed" refusing entry might further jeopardise accrual.

Despite this dilemma, and after a long debate, we have finally recommended the seeking of informed consent in this trial. As a result of our deliberations we have decided that it is now vitally important for the medical profession to brace itself to confront the issue of informed consent squarely, and to examine all its implications with the aim of hammering out an acceptable working ethic for future practice. If doctors, medical researchers, and ethical committees are not prepared to collaborate in a joint initiative of this nature it is all too probable that they will be overtaken by events in the undesirable and potentially extremely damaging shape of a public outcry. Should this happen, emotive and misinformed arguments will inevitably muddy the debate, polarise attitudes, and force the profession into a defensive stance. It then risks being shown as confused and divided on a serious ethical issue which could cause it to suffer a dangerous loss of credibility as well as hampering the prospects for all future clinical trials.

We review the arguments relating to the nature of informed consent, its particular application to randomised clinical trials, and the conclusions arising therefrom. We do not claim to have worked out the final solution to this thorny problem, or even that we have reached a consensus. None the less, we hope that by presenting a summary of our views, others will be encouraged to enter the debate and thus progress towards resolving an ethical code for obtaining informed consent in randomised clinical trials that is acceptable both to the medical profession and to the public at large. 


\section{Informed consent}

To be informed means to be someone who is instructed, who knows the facts. Consent has the following meanings: voluntary agreement, compliance, permission (Concise Oxford Dictionary). It follows from these definitions that if a person is to be in a position to give informed consent to a course of action proposed on his or her behalf by someone else then that person must possess all the relevant facts before giving permission. These facts constitute the "material evidence" necessary to obtain informed consent. If, however, all or part of such evidence is either withheld or distorted then the person being "informed" must be deemed to be involuntarily ignorant, either partially or completely. In such circumstances the person for whom action is proposed has not been enabled to give informed consent.

In philosophical terms one person's right implies another person's obligation and is essentially contractual in nature. The doctor-patient relationship may be said to embody this philosophical concept of contract on two counts. A (the patient) is in a condition requiring medical treatment. $B$ (the doctor) has the expertise. $A$, who has come to $B$, whether privately or through the National Health Service, has the right to $B$ fulfilling the obligation of acting in a certain way towards her, that is to say proposing treatment and care which he considers to be in her best interests. Though $\mathrm{A}$ has surrendered her body to $\mathrm{B}$ for treatment, however, she has not surrendered her right as an individual to self determination. The nature of the contractual relationship between $\mathrm{A}$ and $\mathrm{B}$ therefore imposes on $\mathrm{B}$ the further obligation not to impede that right by withholding any of the material evidence relating to her condition that could (among other things) affect her decision about the treatment proposed. From this it follows that the duty to inform A sufficiently to enable her to give a real and informed consent is an inherent constituent of B's obligation towards A. If $A$ is not fully informed the burden of initiating a dialogue of disclosure rests with $B$, who is informed, and whose duty it is to ensure that the dialogue is opened in such a way that A feels able to take on the burden of responding so that it may then become a continuing dialogue between partners. ${ }^{3}$

Although the contract thus defined is not a legal one, the obligations it imposes on a doctor do have legal implications which are discussed below.

\section{Ethical obligation}

The above definition of informed consent raises several ethical questions in the context of a randomised clinical trial. A patient who has been selected as eligible for entry into a trial cannot know (a) that such a trial is in existence, $(b)$ that her participation is required, $(c)$ the possible benefits and disadvantages of participation, $(d)$ that she has a right to refuse to enter or to withdraw at any stage, and $(e)$ that her selection for a particular type of treatment (or non-treatment) is random, unless she is so informed by her doctors. If any one of these items of knowledge are omitted from the information process is she truly able to give full informed consent ? Furthermore, are doctors entitled to presume that because a patient does not know she therefore does not wish to know? The logic of such a presumption is dubious, let alone the ethics.

Emphatically, doctors are not so entitled according to the Declaration of Helsinki, which states as a basic principle that "in any research on human beings, each potential subject must be adequately (our italics) informed of the aims, methods, anticipated benefits, and potential hazards of the study and the discomfort it may entail. He or she should be informed that he or she is at liberty to abstain from participation in the study and that he or she is free to withdraw his or her consent to participation at any time. The doctor should then obtain the subject's freely given informed consent, preferably in writing." (I.9) 4 Randomisation is not specifically mentioned but is surely implicit in the requirement to inform about methods. Nevertheless, under its guidelines for medical research combined with professional care the declaration also states that "if the doctor considers it essential (our italics) not to obtain informed consent, the specific 3 reasons for this proposal should be stated in the experimental $\stackrel{2}{2}$ protocol for transmission to the independent committee." (II.5) ${ }^{5} \mathrm{C}$

Reasons sufficiently valid to override the ethical obligation to $\widehat{\Omega}$ obtain informed consent are not suggested in the declaration, but clinicians know from experience that there are some circumstances when it could seem "essential" not to obtain informed consent. For example, in a trial of alternative palliative $\varrho$ procedures on patients with advanced carcinoma of the pancreas, full informed consent means that the patient has to be told about a grim prognosis at a stage when the doctor thinks it is wrongo to volunteer this information; yet it could be appropriate for $\frac{}{0}$ full discussion at a later stage. Neither is there any suggestion $\overline{\bar{s}}$ in the declaration of possible sanctions that might be imposed $\stackrel{\varnothing}{\Omega}$ on doctors who refuse to accept an arbitrating body's recommendations in favour of informed consent. This seems correct ${ }^{\text {s }}$ to us, since it is beyond the scope of the declaration to insist on? specific prescriptions for conforming to what are no more than $\vec{\overrightarrow{ }}$ guidelines, or to interfere in the jurisdiction of locally appointed ${ }_{\sigma}$ committees.

Nevertheless, the BMA certainly does not make it easier for doctors, troubled by their consciences, to make up their minds. On the one hand, it emphasises that "the standards as drafted. are only a guide to physicians all over the world"; on the other, it warns that "Doctors are not relieved from criminal, civil, andethical responsibilities under the laws of their own countries."

Brewin recently maintained that much of the confusion about $\vec{\nabla}$ if, when, and from whom it is right to seek informed consento stems from failing to appreciate the distinction between non- $\mathrm{N}$ therapeutic research and randomised treatment. ${ }^{7}$ This is a valid $\triangle$ distinction. From it Brewin argues persuasively that a doctor participating in randomised treatment trials should not be $\overrightarrow{ }$ thought of as a research worker, "but simply as a clinician witho an ethical duty to his patients not to go on giving them treatments. without doing everything possible to assess their true worth."망 Who could quarrel with such a description? But his argument begins to founder when he suggests that this definition of the⿳亠丷厂 doctor's role allows him to dispense with the need to seekô informed consent from trial entrants if, for example, it appears@ too difficult to explain the concept of randomisation, too time $\overrightarrow{\vec{A}}$ consuming, or too alarming.

High ethical standards are required from both the researchers who structure clinical trials and the clinicians who, by volunteer-o ing their participation, explicitly agree to conform to protocol requirements. Patients also have a right to expect equally high ethical standards to apply to the terms of their participation. If informed consent is excluded from the protocol that right is being infringed. To have a right is one matter. Choosing to exercise it it quite another. There will be some (perhaps many) patients who would decline to be fully informed, but this pragmatic consideration cannot modify the intrinsic nature of the right itself. It is? only the person who possesses the right-in this case the patientor - who can decide whether she wishes to exercise it.

Does it therefore follow that the doctor is not allowed to make a judgment, based on his clinical knowledge and experience, that informed consent in certain circumstances is against the best interests of a particular patient? We believe that there are two further distinctions to be drawn that, if properly understood will allow doctors to bridge the gap between conforming to anc ethical imperative and meeting the personal needs of their? patients.

Firstly, there is a vital difference between "seeking" informedo consent and "obtaining" it. The former is an ethical obligation $\mathbb{\mathbb { D }}$ the latter does not necessarily follow, and is not always event possible. Before seeking informed consent a doctor will carefullyo prepare the ground for dialogue, but if, at some stage, the patient states explicitly that she does not wish for further information (although she knows that it is available) and tells the doctor to proceed as he thinks fit, then his duty to inform further ceasese at least while the patient's attitude remains unchanged. In such ${ }^{+}$ 
circumstances, provided that the doctor is satisfied that he is doing the best he can for his patient, which includes making quite clear to her that she can always resume the dialogue, he is entitled to assume his patient's implicit consent.

Secondly, there is a distinction between what constitutes "full" informed consent and what may be regarded as "adequate" information for the purpose of consent. It has been suggested that doctors trying to determine adequacy should be guided by the criterion of what a "reasonable" person would expect to know, that is to say, the degree of material evidence necessary to enable such a person to make a real informed decision. ${ }^{3}$ Schafer maintains that to inform patients that their treatment will be chosen by a randomising formula is part of such material evidence, even though it could be said that since, at the point of entry into the trial no better treatment is known, the information is not relevant to the patient's consent. ${ }^{8} \mathrm{He}$ argues that any information likely to influence the patient's decision (regardless of whether it can be considered rational to be so influenced) must be included. But is it "reasonable" to make a decision based on irrational considerations?

At this point the argument moves from ethics to education. Clearly, ways and means must be found to inform the lay public about the ethical and scientific merits of randomised treatment trials.

\section{Legal implications}

There is no legal precedent in Britain for a patient wishing to bring an action against her doctors for not obtaining her informed consent to participation in a clinical trial. Under the present laws, apparently, such a person has two possible recourses: either an action for battery (when the nature of the procedure has not been defined and has been carried out without obtaining the express consent of the patient) or an action for negligence (when the possible risks have not been explained).

With regard to clinical trials the key question in a case of battery would be whether such definition includes informing the patient that she is participating in a trial. It has been suggested that when the trial is therapeutic (comparing a standard treatment with an alternative) and has been approved by an ethical committee representing "informed medical opinion," consent may "probably be inferred." 9 This seems a weak case, however, when put up against the counter argument that a patient implicitly consents to treatment only on the understanding that her doctor is choosing what he considers to be the best treatment for her. It would follow that if there is doubt in the doctor's mind, and there must be if he is participating in a trial, then he would have an obligation to express that doubt to his patient, and this could include explaining the necessity for random selection. If he does not do so he may be straying outside the terms of his patient's consent.

Nor would it seem to be an adequate defence for a doctor to shift the responsibility for requiring informed consent on to the ethical committees. Ethical committees exist primarily to protect the interests of patients, but this does not mean that they stand in loco parentis, particularly not in circumstances when the individual's rights are being balanced against the demands of scientific research, however laudable. In the reverse situation when a doctor deliberately flouts the ethical committee's requirement to obtain informed consent from the patient he would appear to have seriously weakened his defence.

Somewhat similar arguments could be used in an action for negligence. Negligence is the breach of a duty of care owed by one person to another, in this case the doctor's duty of care to his patient, which includes ensuring that he has told his patient all she needs to know to consent to treatment. If the patient has not been informed that she is in a trial and furthermore has not been warned that there are risks-known or unknown-attached to the experimental treatment for which she has been randomly selected then she could properly say that the doctor had breached his implied obligation towards her (duty of care) by assuming a consent that was not in fact given. Negligence on this basis could be proved, whether or not the patient did indeed suffer serious side effects, but it would not be actionable unless there were some damage.

In the case of a patient entered into a study that is comparing two standard treatments-for example, mastectomy and lumpectomy, as in this trial-if evidence can be produced to show that she had been adequately informed about possible side effects then there could be no grounds for bringing an action in negligence. Nor could a case be brought if, as discussed above, the patient had explicitly refused further information. In law a patient's consent to treatment must count as informed consent if it has been given freely and in the full knowledge that further information is available if required.

British patients are largely unaware that clinical trials are being conducted all over the country; they know even less about how they are conducted, or of their own possible involvement. If we want to avoid the American situation where federal law regulations have made the requirement to obtain informed consent a rigid and somewhat brutal procedure, causing patients to be bombarded with technical details and complicated consent forms so that doctors can comply with the letter of the law and so defend themselves against malpractice suits, it is surely wise to examine these implications now. When the law imposes too heavy a hand it may cause needless anguish.

Under current British law the patient does have adequate means of redress but only if she knows that she has a case to bring. Those doctors who remain unconvinced that they have an ethical obligation to obtain informed consent may be more willing to consider acting from the standpoint of enlightened self interest. The lay public may not know today about randomised clinical trials but in the current climate that state of ignorance is unlikely to persist. It would be a disastrous setback to an evolving relationship between doctor and patient if, on awakening to the existence and requirements of clinical trials, patients and their relatives were to become fearful and hostile, possibly to the extent of embarking on legal action if they believed that in some way advantage had been taken of them.

\section{Doctor's dilemma}

The problems facing doctors who accept informed consent as part of the trial protocol are various and great, and too easily underestimated by the lay public. To begin with, how can the patient's right to determine the type of treatment she is prepared to accept, which must be based on understanding possible medical and social consequences as well as the technical details, ${ }^{10}$ be reconciled with the doctor's duty to act in what he believes to be the best interests of his patient? This dilemma has to be offset against the scientific need to test new treatments offering the potential of better care for future generations of patients. Finally, and most importantly, the doctor has to consider what effect such disclosures will have on the individual patient.

The doctor who wishes to enter patients into a randomised trial can do so only if he honestly does not have a preference for a particular treatment. ${ }^{11}$ It is one thing to admit doubt among one's colleagues, quite another to have to admit it to a patient. In a trial the doctor who seeks informed consent has to admit to his patient that he does not know which is the best treatment; he may have to explain the reasons for random selection. He certainly has to discuss possible side effects, and he may have to disclose more about the prognosis than he would wish. Not unnaturally, a compassionate clinician is concerned that by so taking a patient into his confidence and declaring his own uncertainties this will undermine his patient's trust in him and thus adversely affect the outcome of treatment. Are these fears justified?

To some extent they can be tested only by experience, but evidence suggests that uninformed patients may also be alarmed, anxious, and subject to considerable stress precisely because they are being kept in the dark. ${ }^{12}{ }^{13}$ They may privately suspect that 
bad news is being kept from them. Patients who genuinely do not want to know about their condition may say so frankly, in which case the doctor is relieved of the obligation to make further disclosures. A perceptive doctor will appreciate his patient's insecurities and be concerned to do all he can to improve her understanding of the situation. He should, however, also be aware that sometimes the patient who says, "I leave it to you, doctor, you know best," may be expressing not so much genuine consent as an anxious wish to be compliant and cause as little trouble as possible. To the average patient, the hospital is an awe-inspiring environment where the authority and wisdom vested in doctors is heightened by the deferential attitudes of nursing staff.

Patients who are suffering from a potentially life threatening disease are especially vulnerable, fearful, and subject to influence. Doctors who are enthusiastic about the trial have, therefore, to be on their guard against bringing undue pressures to bear on their patients in an effort to persuade them to participate. Fox and Simpson have cited some examples that clinicians will no doubt recognise. ${ }^{1415}$

\section{Explaining randomisation: the Zelen model}

The major difficulty in explaining randomisation to a patient lies in having to describe alternative treatments before explaining the mechanism of randomisation and the reasons for it (in itself a daunting task). This is confusing for the patient who sees that her doctor does not have control over the choice of treatment and also upsetting if she conceives a preference for one or other; it may well result in her refusal to participate.

Zelen has proposed a new design for randomised trials with the novel feature that before consent is obtained, both patient and doctor know whether an experimental treatment has been assigned. ${ }^{16}$ Eligible patients are randomised into two groups: group $\left(G_{1}\right)$ is called "do not seek consent" which means that they are not asked for their consent to enter the clinicial trial and they receive the best standard treatment (A); patients randomised to group $\left(G_{2}\right)$ are asked for their full informed consent to participate in the trial and receive the experimental treatment (B). If they do not agree to receive treatment $(B)$ then they receive treatment $(\mathrm{A})$.

The obvious advantage in this design is that the doctor has only to discuss a single treatment with his patient and so will not appear compromised because he is uncertain and apparently subjecting his patient to "a flick of the computer switch." The patient is spared the need to agree in advance to a randomised allocation of treatment.

Gloriously simple though it is, Zelen admits that it does raise new ethical problems. In this present context the first is the most important-namely, certain patients (group $G_{1}$ ) are still being randomised and entered into a trial without giving their informed consent. To argue that they are receiving the best standard treatment that they have a right to expect, therefore there is no need to inform them about an alternative experimental procedure and, further, that as members of a control group they will be exceptionally well managed (a reassurance for doctors rather than patients who expect it anyway) smacks of justification, not strict ethical reasoning.

\section{Informed consent in relation to a breast conservation trial}

The attraction of adopting the Zelen model in a breast conservation trial is obvious. Women confronted with the choice between two types of surgery, one more mutilating than the other, will, as Schafer clearly outlines, have different priorities, depending on their concept of self and self image. ${ }^{8}$ For some the putative improvement in survival at whatever aesthetic expense will be of supreme importance; for others, preserving their breast will matter more than uncertainty about mortality; and in between there will be various intermediate positions depending on the trade offs the individual makes between risks on the one hand and benefits and harms on the other. The emotional stress induced by this particular disease is profound; inevitably it will be increased if patients are first told the alternatives and then informed that their selection will be random. On the Zelen plan only those women randomised for lumpectomy (treatment B) would be asked for their informed consent. Those randomised for mastectomy (treatment A) would not be informed that they were in a trial.

The working party considered this option at length and eventually rejected it on the grounds that it was unethical to seek full informed consent from only one of the groups. It also considered the possibility (faint but not inconceivable) that a patient receiving a mastectomy who later discovered that she had been entered into in a trial without her knowledge and had thus perhaps been denied the chance to opt for the more conservative procedure, would consider this cause for legal action.

How best to explain randomisation and seek full informed consent was not resolved, and the working party's final recommendation was that in this trial informed consent is desirable but not obligatory. Participating surgeons are advised that it is essential to submit the protocol to their local ethical committee, which will then advise on the need for informed consent.

\section{Ethical consequences of not doing randomised controlled clinical trials}

We have concerned ourselves predominantly with the ethical, legal, and medical implications of seeking informed consent from patients entering randomised clinical trials. It must be emphasised that these same problems exist in the absence of randomised clinical trials. There is a long catalogue of disasters that have followed the enthusiastic introductions of new surgical and medical techniques without the benefit of proper experimental method. ${ }^{17-19}$ For that reason, when any new treatment is introduced the first group of patients to receive it needs to be handled in the same way as patients entering into controlled clinical trials. Where the dilemma is then magnified becomes apparent when considered from two additional points of view.

The proponents of any new treatment are naturally enthusiastic and commonly have an almost total and uncritical belief in their new ideas. It would, therefore, be easy for them to persuade patients to accept this new treatment, particularly if they were suffering from a potentially fatal disease and had exhausted conventional treatment, even though the new treatment might impair the length or quality of survival. Furthermore, as a result of either systematic or random bias, the results of treatment from uncontrolled studies, carried out by enthusiasts, almost always look encouraging, and therefore a position is rapidly reached when the clinicians concerned begin to think it is unethical to withhold the new operation or wonder drug. As a result, it may take anything up to 30 years for the unfortunate side effects to become recognised ${ }^{18}$ or before careful and critical scrutiny shows that any apparent benefit may be ascribed to selection or the placebo effect. ${ }^{19}$ The additional ethical and medical implications of uncontrolled experimentation are that harmful side effects are recognised much later than in controlled experimentation.

It could be argued forcibly that if the climate of opinion concerning informed consent changes in this nation over the next few years, so that patients entering random controlled trials must of necessity give full informed consent, then the same standards should apply to patients in uncontrolled experiments. This argument may be taken to its logical conclusion: that clinicians treating patients outside any protocol in any area of controversy also have the obligation to inform their patients of the alternative treatments that are being offered in different parts of the country at the same time.

This paper concerns value conflicts. The principal value conflict that we are discussing is between details of patient care 
and progress in medical science. It should be remembered that other value conflicts exist for patients being treated outside any experimental protocol-for example, care of the patient against care of the pocket.

\section{Practical proposals for improving procedures for seeking informed consent}

(1) Eligible patients should be given the option to take time to consider giving their consent, perhaps along the lines described by Simpson at the Wellington Hospital in Australia. ${ }^{15}$ Here the patient is fully informed about the trial by her physician or surgeon but an informal consent in principle only is obtained. At a later date the procedures are again explained and only then is formal consent obtained by asking her to sign a consent form.

(2) The consent form should be fairly non-specific but it must be backed up by as much verbal explanation as possible. Signature to such a form in the presence of a witness might have legal validity if it included the phrase "the effect and nature of such treatment have been explained to me," but only if it could be proved that the explanation had been given. ${ }^{9}$

(3) Ideally, a trained nurse counsellor or other suitably qualified person should help to obtain informed consent, and the patient should be made aware that she may resume this continuing dialogue at any time.

(4) Ethical committees should view the issue of informed consent as a top priority, bearing in mind its various applications -in the ordinary clinical situation, in therapeutic trials, and in experimental research. They should reconsider the type of guidelines to propose to doctors, with reference to the Declaration of Helsinki and other national and international codes and regulations; they should consider practical ways of improving consent procedures in their hospitals; and they should monitor these procedures, perhaps by requesting reports at stated intervals.

(5) Those doctors who treat patients with cancer but do not participate in randomised clinical trials should realise that they too have an obligation to discuss alternative forms of treatment with their patients. In our view the fact that they are not formally randomising their patients does not reduce their obligation in this respect.

\section{Conclusion}

Randomised clinical trials are the most efficient scientific means of comparing alternative treatments. They are also the most ethical. It is our view that doctors have an ethical obligation to seek informed consent from patients who are prospective entrants into the breast conservation trial. In clinical trials generally, the issue of informed consent remains unresolved. It is intolerable, however, that the burden of accountability should be placed entirely on doctors while there remains this uncertainty. It is, therefore, a matter for urgent concern, and action, that doctors should be helped to formulate a workable code of conduct for all clinical trials by ethical committees consisting of informed lay members as well as doctors. The cooperation of other bodies concerned to advance scientific knowledge in the cause of improving current medical and surgical treatments for patients with cancer should also be sought. In the absence of such action clinicians will be frightened off joining in such studies, and patients will be frightened away from entering the trials. As a result all the efforts of the past 20 years in establishing the random controlled trial as the preferred scientific method for evaluating new treatment will be nullified, taking us back into the dark ages where therapeutic innovations were judged by intuition and wishful thinking.

\section{References}

${ }^{1}$ Bradford Hill A. Medical ethics and controlled trials. Br Med f 1963;ii : 1043-9.

${ }^{2}$ Peckham MJ (Chairman). Protocol for a collaborative trial to evalute the need for mastectomy in the management of early breast cancer. London: Cancer Research Campaign Clinical Trials Centre, 1982.

${ }^{3}$ Kraft RB. The breast cancer controversy and its implications for the informed consent doctrine. Fournal of Legal Medicine 1980;2:47-84.

${ }^{4}$ British Medical Association. The Handbook of Medical Ethics. London: BMA, $1981: 68$

${ }^{5}$ British Medical Association. The Handbook of Medical Ethics. London: BMA, 1981:69.

- British Medical Association. The Handbook of Medical Ethics. London: BMA, $1981: 67$

${ }^{7}$ Brewin TB. Consent to randomised treatment. Lancet 1982 ;ii :919-21.

${ }^{8}$ Schafer A. The ethics of the randomized clinical trial. $N$ Engl $f$ Med $1982 ; 307: 719-24$

${ }^{9}$ Elkeles A. Informed consent in clinical trials. Lancet $1982 ; \mathrm{i}: 1189$.

10 Somerville M. Consent to medical care. Ottawa: Law Commission of Canada, 1979. (Study paper prepared for the Law Reform Commission of Canada: protection of life series.)

${ }^{11}$ Peto R, Pike MC, Armitage P, et al. Design and analysis of randomised clinical trials requiring prolonged observation of each patient. 1 Introduction and design. $B r \mathcal{F}$ Cancer 1976;34:585-612.

${ }^{12}$ Maguire P. The psychological and social sequelae of mastectomy. In: Howells J, ed. Modern perspectives in the psychiatric aspects of surgery. New York: Brunner-Muzel, 1976:390-421.

${ }^{13}$ Hinton J. Comparison of places and policies for terminal care. Lancet 1979 ; i :29.

14 Fox RM. Situations creating ethical stress. Med $\mathcal{f}$ Aust 1981;1:162-3.

15 Simpson JS. Informed consent: a medical viewpoint. Med f Aust 1981;1: 169-71.

16 Zelen M. A new design for randomized clinical trials. $N$ Engl $f$ Med 1979;300:1242-5.

${ }^{17}$ Baum M. Scientific empiricism and clinical medicine. $\mathcal{F} R$ Soc Med 1981; 74:504-9.

18 Barnes BA. Discarded operations: surgical innovation by trial and error. In: Bunker JP, Barnes BA, Mosteller F, eds. Costs, risks and benefits of surgery. New York: Oxford University Press, 1977:109-23.

19 Altman DG. Statistics and ethics in medical research. In: Gore SM, Altman DG, eds. Statistics in practice. London: British Medical Association, 1982:1-26.

(Accepted 8 February 1983)
Is curvature of the erect penis a common phenomenon? Does it tend to get worse, and if so is there any treatment?

Congenital curvature of the penis on erection or chordee is usually associated with hypospadias or, more rarely, a congenitally short urethra or unequally developed corpora. Acquired angulation sometimes follows trauma where there has been damage to one of the corpora cavernosa, but the commonest condition causing curvature of the erect penis is Peyronie's disease, which is not infrequently seen as a clinical problem in urological practice. In this condition a dense, irregular fibrous plaque of tissue develops between Buck's fascia and the corpora cavernosa. This distorts and angulates the penis on erection. Often this is associated with pain in the initial stages but this eventually subsides, though impotence often results. The condition tends to regress spontaneously so evaluation of the large variety of medications used for treatment is difficult. Surgery should be deferred to allow for the possibility of spontaneous improvement and should not be undertaken if sexual intercourse is possible. If the curvature or angulation is too great to allow penetration then Nesbit's ${ }^{1}$ operation should be considered. In this operation an ellipse is excised from the corpora opposite the point of maximum curvature to straighten the penis at the expense of length. The insertion of silicone rods into the corpora is appropriate if the patient is also impotent. ${ }^{2}-$ J C GINGELL, consultant urologist, Bristol.

' Nesbit RM. Congenital curvature of the phallus: report of three cases with

description of corrective operation. $\mathcal{F}$ Urol $1965 ; 93: 230-2$.
2 Jonas U, Jacobi GH. Silicone-silver penile prosthesis: description, operative approach and results. $\mathcal{F}$ Urol $1980 ; 123: 865-7$. 